\title{
Tracing the evolution of the symmetry energy of hot nuclear fragments from the compound nucleus towards multifragmentation
}

\author{
G. A. Souliotis ${ }^{1}$, A. S. Botvina ${ }^{2}$, D. V. Shetty ${ }^{1}$, A. L. Keksis ${ }^{1}$, M. Jandel ${ }^{1}$ \& M. Veselsky ${ }^{1}$ 的 and S. J. Yennello ${ }^{1}$ \\ ${ }^{1}$ Cyclotron Institute, Texas A $8 M$ University, College Station, TX 77843 and \\ 2 Institute for Nuclear Research, Russian Academy of Sciences, RU-117312 Moscow, Russia
}

(Dated: September 22, 2018)

\begin{abstract}
The evolution of the symmetry energy coefficient of the binding energy of hot fragments with increasing excitation is explored in multifragmentation processes following heavy-ion collisions below the Fermi energy. In this work, high-resolution mass spectrometric data on isotopic distributions of projectile-like fragments are systematically compared to calculations involving the Statistical Multifragmentation Model (SMM). Within the SMM picture, the present study suggests a gradual decrease of the symmetry energy coefficient of the hot primary fragments from $25 \mathrm{MeV}$ at the compound nucleus regime towards $15 \mathrm{MeV}$ in the multifragmentation regime. The isotopic distributions of the hot primary fragments are found to be very wide and extend towards the neutron drip-line. These findings are expected to have important implications to the modeling of the composition and the evolution of hot and dense astrophysical environments, such as those of core-collapse supernova.
\end{abstract}

PACS numbers: 25.70.-z, 25.70.Hi,25.70.Lm,26.30.+k

Nuclear multifragmentation is one of the most interesting phenomena in nuclear physics as it holds promise for understanding nuclear matter properties at the extreme conditions of high excitation energy and large isospin $(N / Z)$ asymmetry [1, 2, 3, $, 4,[5]$. The latter, in particular, plays a profound role in the dynamics of various astrophysical environments $[6,7,6,8,9]$. It is well established (e.g. 4]) that nuclear systems with relatively low excitation energy $\left(E^{*} / A \leq 2 \mathrm{MeV}\right)$ form the traditional compound nucleus, whereas at higher excitation energy, the hot nuclear system expands and, subsequently, disassembles into an ensemble of hot primary fragments. This extremely complicated process, namely the multifragmentation, occurs on a timescale of $100 \mathrm{fm} / \mathrm{c}\left(3.3 \times 10^{-22} \mathrm{sec}\right)$ during which the system can sample a large number of configurations. For this reason, statistical calculations (e.g., [10, 11]) have been very successful in describing this process.

Recently, a remarkable similarity has been pointed out between the thermodynamic conditions (temperature, density, isospin asymmetry $N / Z$ ) reached in nuclear multifragmentation and the collapse/explosion of massive stars [12, 13, 14]. This observation opens up the possibility of applying well-established models of nuclear reactions to describe matter distribution and evolution during supernova explosions [12]. In addition, statistical calculations suggest that in multifragmentation 12, 15 and in hot astrophysical environments (e.g. supernova) [12, 16], the ensemble of primary fragments includes neutron-rich nuclei towards or beyond the neutron drip-line.

The primary fragments are expected to be hot (with excitation energies approaching $2-3 \mathrm{MeV} /$ nucleon [17]) and, initially, in close proximity to neighboring fragments or nucleons. These conditions render their properties, e.g. binding energy, different from those of cold (ground state) isolated nuclei. In particular, recent studies [18, 19, 20, 21] give evidence for a significant decrease of the symmetry energy of hot primary fragments. We recall that for a system with $N$ neutrons and $Z$ protons, the symmetry energy can be expressed as $E_{\mathrm{sym}}=$ $C_{\text {sym }}(N-Z)^{2} / A$, with $C_{\text {sym }}$ the symmetry energy coefficient and $A$ the mass number. In the aforementioned studies, the symmetry energy of hot fragments from multifragmentation at high excitation energy $\left(E^{*} / A=4-6\right.$ $\mathrm{MeV}$ ) is found to be reduced to $C_{\mathrm{sym}} \sim 15 \mathrm{MeV}$ or lower, as compared to the conventional value of $C_{\text {sym }} \sim 25 \mathrm{MeV}$ for cold nuclei. In the same vein, our recent work 22] indicated decreased symmetry energy in the region $E^{*} / A$ $=2-3 \mathrm{MeV}$. The observed reduction of the symmetry energy implies that more neutron-rich nuclei are favored in the distribution of fragments after the partitioning of the initial hot and expanded nuclear system. Such unusual neutron-rich nuclei should also be quite abundant in supernova matter [12] and may affect the dynamics of the explosion and the subsequent nucleosynthesis.

For completeness, we emphasize that presently there is strong and growing interest in the density dependence of the symmetry energy of nuclear matter (e.g. [1, 9, 23, 24] and references therein). However, we should point out that at low densities (less than half of the normal nuclear density) the uniform nuclear matter breaks-up into fragments. Thus, in order to describe hot fragment distributions, we should address the problem of the symmetry energy of individual fragments (primary fragments) embedded in a hot and dense environment.

The present Rapid Communication is intended to provide evidence for a continuous evolution of the symmetry energy coefficient from the compound nucleus regime $\left(E^{*} / A \leq 2 \mathrm{MeV}\right)$ towards multifragmentation $\left(E^{*} / A \geq 4\right.$ $\mathrm{MeV}$ ) by a systematic comparison of the isotopic distributions of heavy fragments with calculations based on the 
Statistical Multifragmentation Model (SMM). Herein, fragments with $A>40$ are referred to as heavy residues, those with $A<40$ as heavy IMF (intermediate mass fragments), whereas both groups are collectively called heavy fragments. Moreover, in this work, the $N / Z$ distributions of hot nuclei from the multifragmentation of neutron-rich systems are found to be very wide involving exotic nuclei towards the neutron drip-line.

The experimental data were obtained at the Cyclotron Institute of Texas A\&M University employing beams from the K500 Superconducting Cyclotron. Two magnetic separators were used: the Momentum Achromat Recoil Separator (MARS) for the ${ }^{86} \mathrm{Kr}(25 \mathrm{MeV} /$ nucleon) $+{ }^{64} \mathrm{Ni},{ }^{124} \mathrm{Sn},{ }^{208} \mathrm{~Pb}$ reactions and the Superconducting Solenoid Line (BigSol) for the ${ }^{64} \mathrm{Ni}(25 \mathrm{MeV} /$ nucleon $)+$ ${ }^{64} \mathrm{Ni}$ reaction. A detailed description of the measurements is presented in 22, 25, 26]. Employing standard $B \rho-T O F-\triangle E-E$ (magnetic rigidity, time-of-flight, energy loss and residual energy, respectively) techniques [26], the atomic number $Z$, the mass number $A$ and the velocity of the fragments were obtained with high resolution. The measurements of ${ }^{86} \mathrm{Kr}+{ }^{64} \mathrm{Ni},{ }^{124} \mathrm{Sn}$ were performed in the angular range $2.7^{\circ}-5.4^{\circ}$ and $B \rho$ range $1.3-2.0 \mathrm{~T} \mathrm{~m}$. The measurements of the ${ }^{86} \mathrm{Kr}+{ }^{208} \mathrm{~Pb}$ were obtained in the angular range $1.0^{\circ}-2.7^{\circ}$ and $B \rho$ range $1.2-1.7 \mathrm{~T} \mathrm{~m}$. Finally, the ${ }^{64} \mathrm{Ni}+{ }^{64} \mathrm{Ni}$ measurements were performed with BigSol in the angular range $1.5^{\circ}-3.0^{\circ}$ and $B \rho$ range 1.1-1.6 T m 22]. The measured yield distributions were used to extract the average $Z / A$ values. To obtain total fragment cross sections, the measured yield data were corrected for the spectrometer acceptance (as in 26]) with the aid of the simulations described below.

The calculations are based on a two-stage Monte Carlo approach. The dynamical stage of the collision was described by the deep-inelastic transfer (DIT) code of Tassan-Got 27] simulating stochastic nucleon exchange in peripheral and semiperipheral collisions. This model has been successful in describing the isospin, excitation energy and kinematical properties of excited quasiprojectiles in a variety of recent studies [26, 28, 29, 30]. The deexcitation of the quasiprojectiles was performed with the latest version of the Statistical Multifragmentation Model (SMM) [10, 14, 31], referred to as "SMM05" and briefly summarized below. This model assumes statistical equilibrium at a low-density freeze-out stage and includes all breakup channels ranging from the compound nucleus to vaporization (channels with only light particles $A<4$ ), allowing a unified description of nuclear disintegration with increasing excitation. In the microcanonical treatment, the statistical weight of a decay channel is calculated as exponential of the entropy. Light fragments with $A<4$ are considered as stable particles (nuclear gas) with only translational degrees of freedom. Fragments with $A>4$ are treated as heated liquid drops with free energies parameterized as a sum of volume, surface, Coulomb and symmetry energy terms [14] with pa- rameters adopted from the Bethe-Weizsacker mass formula. The model generates a Markov chain of partitions (with the Metropolis algorithm) representative of the whole partition ensemble [31]. In the freeze-out configuration, the hot primary fragments are assumed to be isolated and at normal density; they, subsequently, propagate in their mutual Coulomb field, while undergoing binary deexcitation via evaporation, fission or Fermi breakup [10]. The Coulomb interaction energy is directly calculated for each spatial configuration of fragments in the freeze-out volume. Finally, the effect of the Coulomb field of the target in proximity to the decaying quasiprojectile is included. The symmetry energy evolution is taken into account in the mass calculation of the primary fragments and the secondary fragments during the deexcitation stage, as in [14]. Below a threshold of $E^{*} / A=1 \mathrm{MeV}$, a smooth transition to standard experimental masses is assumed [14]. Standard parameters of the SMM code are employed. A multiplicity dependent parametrization of the free volume [10] (determining the contribution of the fragment translational motion to the partition probability [10]) is used, whereas, the freeze-out volume (determining the Coulomb energy of the fragment partition) is taken to be 6 times the nuclear volume at normal density (as suggested by the asymptotic velocities of the observed heavy fragments).

Fig. 1 shows the average proton fraction $\langle Z / A\rangle$ of the fragments with respect to mass for the reactions presented in order of increasing system $N / Z$. The experimental data are shown by solid symbols and span the whole range of masses from evaporation residues down to heavy IMF. Vertical arrows indicate an approximate separation between heavy residues from evaporation and from the onset of multifragmentation. The thin solid line (marked "SL") gives the line of $\beta$ stability and the thin dotted line (marked "EAL") represents the evaporation attractor line [32] (corresponding to the locus of fragment yields produced by evaporation of neutrons and charged particles from highly excited nuclei close to stability). The evaporation residue regime is populated mostly by neutron deficient products, except for the heaviest fragments from ${ }^{86} \mathrm{Kr}+{ }^{64} \mathrm{Ni}$ and ${ }^{86} \mathrm{Kr}+{ }^{124} \mathrm{Sn}$ corresponding to very peripheral products 25]. Fragments on the left side of the arrow (produced above the multifragmentation threshold of $E^{*} / A \sim 2 \mathrm{MeV}$ ) show progressively smaller values of $Z / A$ (Fig. 1) and cross the line of $\beta$ stability.

The results of the DIT/SMM05 calculations are presented by thick lines. The dotted lines correspond to calculations with constant values of the symmetry energy of $C_{\mathrm{sym}}=25,20,15 \mathrm{MeV}$ from the upper to the lower line, respectively. We observe that the standard calculation with $C_{\text {sym }}=25 \mathrm{MeV}$ produces fragments that are, on average, more neutron deficient than the observed fragments. With $C_{\text {sym }}=20 \mathrm{MeV}$, the agreement with the data seems to improve, except for the lowest 


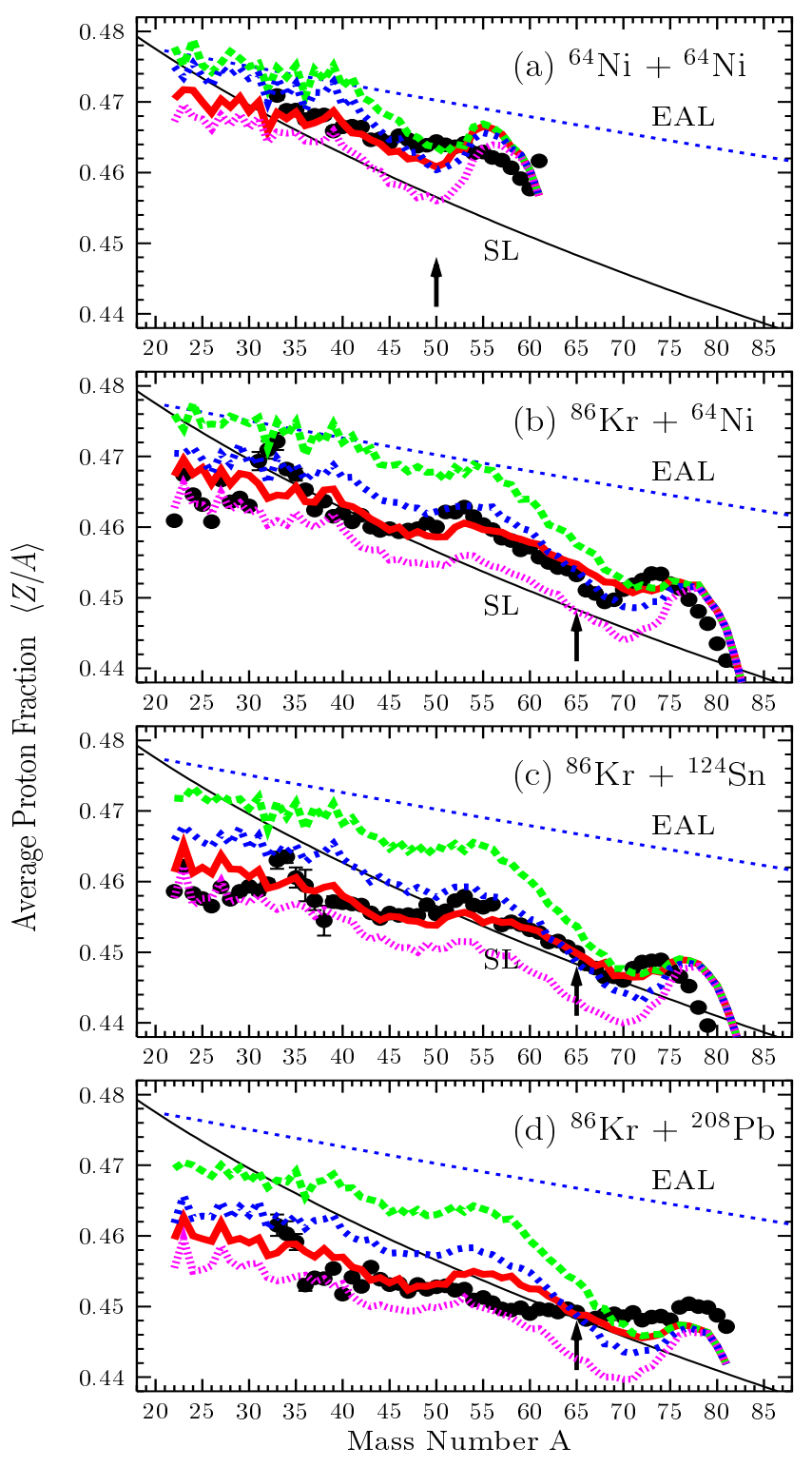

FIG. 1: (Color Online) Average Z/A vs A. (a) ${ }^{64} \mathrm{Ni}(25 \mathrm{MeV} /$ nucleon $)+{ }^{64} \mathrm{Ni}$ (BigSol data). (b), (c), (d) ${ }^{86} \mathrm{Kr}(25 \mathrm{MeV} /$ nucleon $)+{ }^{64} \mathrm{Ni},{ }^{124} \mathrm{Sn},{ }^{208} \mathrm{~Pb}$, respectively (MARS data). Solid points: data. Thin solid line (SL): line of stability. Thin dotted line (EAL): evaporation attractor line. Thick lines: DIT/SMM05 calculations. Dotted lines, top to bottom: with $C_{\text {sym }}=25,20,15 \mathrm{MeV}$, respectively. Thick solid line: with $C_{\mathrm{sym}}\left(E^{*} / A\right)$ given by the thick line in Fig. 2 . Arrows: multifragmentation vs compound nucleus regime.

masses, whereas with $C_{\text {sym }}=15 \mathrm{MeV}$, only the latter group of masses is described. We point out that the measured heavy fragment data at $25 \mathrm{MeV} /$ nucleon span a continuous range of excitation energies up to $E^{*} / A=$ 4.0-4.5 MeV (corresponding to $\sim 95 \%$ of all produced fragments). Assuming a continuous behavior of the symmetry energy from low to high excitation, we tested various forms of the dependence of $C_{\text {sym }}$ on excitation energy $E^{*} / A$. We found that the form represented by the thick solid line in Fig. 2, referred to as form "2-4" in the following, [assigning $C_{\text {sym }}=15 \mathrm{MeV}$ to events with $\left.E^{*} / A>4 \mathrm{MeV}\right]$ provides a remarkable agreement of the calculations with the data from multifragmentation-like processes, as shown by the solid lines in Fig. 1.

The thin solid lines and the thin dashed line in Fig. 2 indicate three additional forms of $C_{\mathrm{sym}}\left(E^{*} / A\right)$ that we tested. The results of $\langle Z / A\rangle$ for $\mathrm{Kr}+\mathrm{Sn}$ are shown in Fig. 3 by the corresponding thin lines (the lower one corresponding to the left thin line of Fig. 2 and the upper one to the right thin line). In Fig. 3, we notice that the thin solid lines embrace the thick line obtained with the "2-4" form of $C_{\text {sym }}\left(E^{*} / A\right)$, whereas the thin dashed line is almost indistinguishable from the thick one. Similar results are obtained for the other reactions of this work. Furthermore, assuming that the multifragmentation threshold is at $E^{*} / A=2 \mathrm{MeV}$, we may only adopt forms of $C_{\text {sym }}\left(E^{*} / A\right)$ with the condition $C_{\text {sym }}=25 \mathrm{MeV}$ at the threshold and below. An appropriate such form appears to be the "2-4" form represented by the thick solid line in Fig. 2, as mentioned previously. Consequently, within the present SMM interpretation of fragment formation, the symmetry energy coefficient appears to decrease from $25 \mathrm{MeV}$ in the compound nucleus regime (left arrow in Fig. 2) to $15 \mathrm{MeV}$ in the bulk multifragmentation regime (right arrow in Fig. 2). This phenomenological evolution of the symmetry energy of hot heavy fragments is the main result of the present study.

For comparison, in Fig. 2 we include the values of the symmetry energy coefficient obtained in our recent work on heavy fragment isoscaling from the ${ }^{86} \mathrm{Kr}$ (circle, square) and ${ }^{64} \mathrm{Ni}$ (triangle) reactions [22]. We notice that, in spite of the different approach of symmetry energy determination, an overall agreement in the trend is observed. We speculate that the reduction of the symmetry energy with excitation observed in this work and the aforementioned previous studies [18, 19, 20, 21] may originate from in-medium modifications of the properties of the hot primary fragments in their dense surrounding [33, 34]. Detailed microscopic calculations are necessary to shed light on this interesting and challenging issue [34].

Apart from the average $Z / A$ properties, the present calculations describe successfully the characteristics of the observed fragments including the widths of the isotopic mass distributions, the velocity distributions and the production cross sections. A full report on these results is currently underway. For completeness, we present in Fig. 4 a comparison of the experimental mass distributions for several elements with the DIT/SMM05 calculations for two reactions: ${ }^{86} \mathrm{Kr}+{ }^{64} \mathrm{Ni}$ and ${ }^{86} \mathrm{Kr}+{ }^{208} \mathrm{~Pb}$. The "2-4" form of $C_{\text {sym }}\left(E^{*} / A\right)$ dependence (thick line in Fig. 2) was used in these calculations. For $\mathrm{Kr}+\mathrm{Ni}$, the data are shown by the full circles and the calculations by the thick solid lines (the thin solid lines giving the hot primary fragment distributions for these elements). Similarly, for $\mathrm{Kr}+\mathrm{Pb}$, the data are given by 


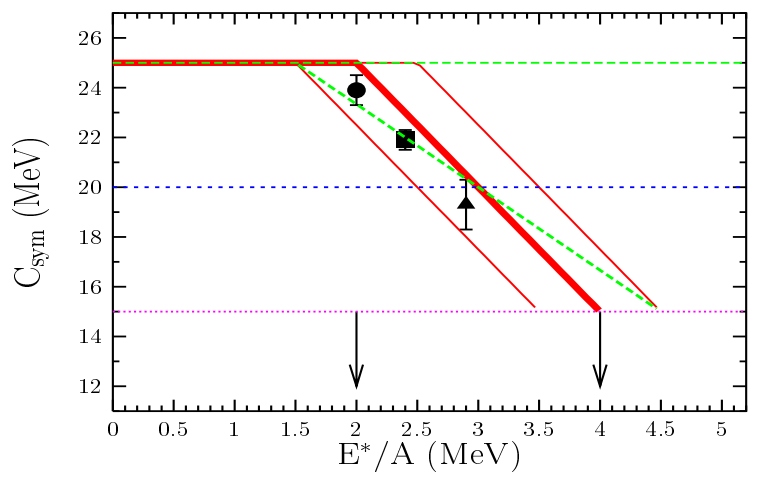

FIG. 2: (Color online) Thick solid line: form "2-4" of $C_{\text {sym }}\left(E^{*} / A\right)$ in the SMM05 calculations. Thin lines: other forms of $C_{\mathrm{sym}}\left(E^{*} / A\right)$ tested (see text and Fig. 3). Thin horizontal lines (top to bottom): $C_{\text {sym }}=25,20,15 \mathrm{MeV}$, respectively, used in Fig. 1 calculations. Solid points: values of $C_{\text {sym }}$ from [22].

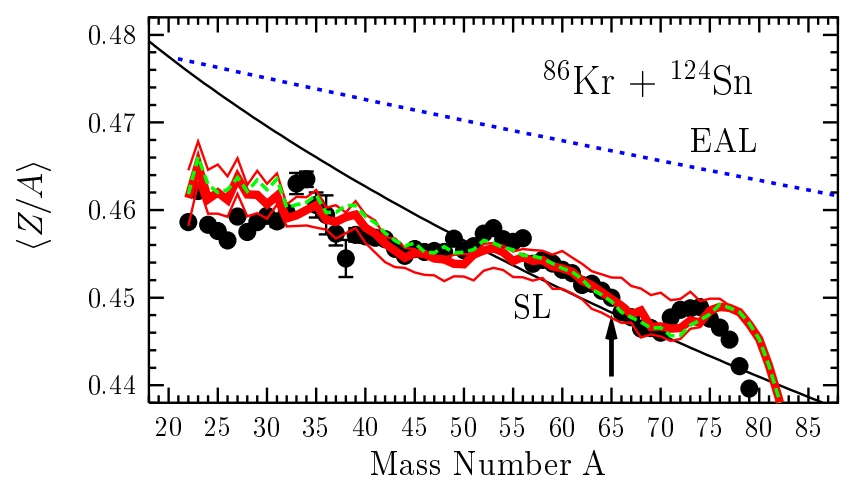

FIG. 3: (Color Online) $\langle Z / A\rangle$ vs A for ${ }^{86} \mathrm{Kr}+{ }^{124} \mathrm{Sn}$. Solid points (data), lines SL, EAL and arrow as in Fig. 1. Thin lines tracing the data: DIT/SMM05 calculations with $C_{\text {sym }}\left(E^{*} / A\right)$ forms presented by the respective lines in Fig. 2 (see text).

open circles and the calculations by thick dotted lines (again, the thin dotted lines describing hot fragments). We find an overall satisfactory agreement of the calculations with the mass distribution data. Interestingly, the $\mathrm{Kr}+\mathrm{Pb}$ data extend to more neutron-rich products than those of $\mathrm{Kr}+\mathrm{Ni}$ (demonstrating the significant role of the target $N / Z$ at this energy regime 25,30$]$ ), a trend well described by the calculation. Finally, we point out that the distributions of the hot primary fragments are wide and include very neutron-rich nuclei. Particularly, for the $\mathrm{Kr}+\mathrm{Pb}$ reaction (the most neutron-rich system studied), they are very broad and extend towards the neutron drip-line 35] (arrows in Fig. 4). Our calculations indicate that the distributions of these hot exotic nuclei are sensitive to their masses and, in turn, sensitive to the evolution of the symmetry energy with excitation.

We wish to emphasize that high-resolution mass spectrometric data on heavy fragment (heavy residue and heavy IMF) distributions - spanning the full spectrum of
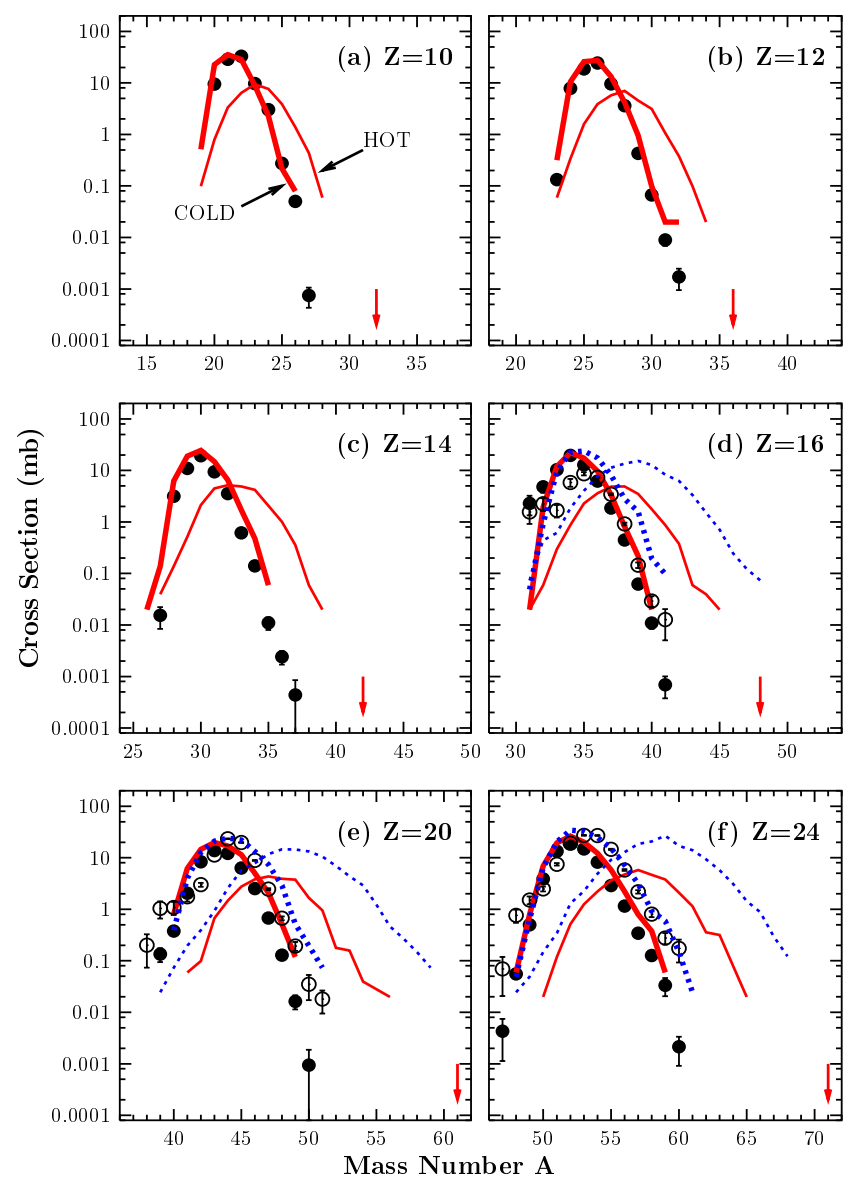

FIG. 4: (Color online) Comparison of experimental mass distributions with DIT/SMM05 calculations. Solid points: ${ }^{86} \mathrm{Kr}+{ }^{64} \mathrm{Ni}$ data. Open points: ${ }^{86} \mathrm{Kr}+{ }^{208} \mathrm{~Pb}$ data. Full lines: calculations for ${ }^{86} \mathrm{Kr}+{ }^{64} \mathrm{Ni}$; thick: final (cold) fragments, thin: primary (hot) fragments. Dotted lines: calculations for ${ }^{86} \mathrm{Kr}+{ }^{208} \mathrm{~Pb}$; thick: final (cold) fragments, thin: primary (hot) fragments. Arrows: neutron-drip line [35].

reaction mechanisms (and excitation energies) from the traditional compound nucleus towards multifragmentation - may provide a promising experimental probe of the properties of hot and extremely neutron-rich fragments when coupled with detailed calculations as presented in this study. From an experimental point of view, we wish to propose systematic measurements on residue distributions on a variety of neutron-rich systems. The energy regime of $\sim 25 \mathrm{MeV} /$ nucleon has two main advantages: (a) $N / Z$ equilibration is attained (e.g. [30]), thus the target has maximum contribution to the $N / Z$ distribution of the quasiprojectiles and (b) the excitation energy is not too high $\left(E^{*} / A<4 \mathrm{MeV}\right)$, so that hot heavy primary fragments are abundantly present. A very exciting extension of these studies in the future involves reactions with very neutron-rich rare beams on heavy neutron-rich targets in current or planned radioactive beam facilities (e.g. the Advanced Exotic Beam facility in the US).

We believe that comparisons of high-resolution heavy 
fragment data with precise model calculations can provide valuable information on the distributions and the properties of hot and very neutron-rich nuclei towards and beyond the neutron drip-line. Knowledge on the properties of exotic nuclei produced in hot and dense environments are essential for astrophysical calculations, such as the composition and dynamics of core-collapse supernova and the course of the relevant nucleosynthesis processes (e.g., the r process).

In summary, experimental data on isotopic distributions of projectile-like fragments from peripheral and semiperipheral collisions of $25 \mathrm{MeV} /$ nucleon ${ }^{86} \mathrm{Kr}$ and ${ }^{64} \mathrm{Ni}$ beams on heavy targets are compared to calculations involving the Statistical Multifragmentation Model (SMM). Within the adopted SMM picture, the present study suggests a gradual decrease of the symmetry energy coefficient of the hot primary fragments from 25 $\mathrm{MeV}$ in the compound nucleus regime towards $15 \mathrm{MeV}$ in the multifragmentation regime. The isotopic distributions of the hot fragments following the multifragmentation stage are very broad and, for the most neutron-rich systems, are found to extend to exotic neutron-rich nuclei. These findings are of importance in calculations of the isotopic composition in hot and dense astrophysical environments, such as those encountered in supernova.

We are thankful to L. Tassan-Got for the DIT code. We gratefully acknowledge the support of the Cyclotron Institute staff during the measurements. Financial support for this work was provided, in part, by the U.S. Department of Energy under Grant No. DE-FG0393ER40773 and by the R.A. Welch Foundation under Grant No. A-1266. A.S.B was supported in part through grant RFFR 05-02-04013 (Russia) and M.V. through VEGA-2/5098/25 (Slovakia).

* C-INC, Los Alamos National Laboratory, Los Alamos, NM 87545.

† Institute of Physics of the Slovak Academy of Sciences, Bratislava, Slovakia.

[1] V. Baran, M. Colonna, V. Greco, and M. DiToro, Phys. Rep. 410, 335 (2005).

[2] P. Chomaz, M. Colonna, and J. Randrup, Phys. Rep. 389, 263 (2004).

[3] P. Danielewicz, R. Lacey, and W. G. Lynch, Science 298,
1592 (2002).

[4] J. Richert and P. Wagner, Phys. Rep. 350, 1 (2001).

[5] L. G. Moretto, R. Ghetti, L. Phair, K. Tso, and G. J. Wozniak, Phys. Rep. 287, 249 (1997).

[6] A. W. Steiner, M. Prakash, J. M. Lattimer, and P. J. Ellis, Phys. Rep. 411, 325 (2005).

[7] J. M. Lattimer and M. Prakash, Science 304, 536 (2004).

[8] S. Woosley and T. Janka, Nature Physics 1, 147 (2005).

[9] B. A. Li and W. U. Schröder, eds., Isospin Physics in Heavy Ion Collisions at Intermediate Energies (Nova Science, New York, 2001).

[10] J. P. Bondorf, A. S. Botvina, A. S. Iljinov, I. N. Mishustin, and K. Sneppen, Phys. Rep. 257, 133 (1995).

[11] D. H. E. Gross, Rep. Prog. Phys. 53, 605 (1990).

[12] A. S. Botvina and I. N. Mishustin, Phys. Lett. B 584, 233 (2004).

[13] A. S. Botvina and I. N. Mishustin, Phys. Rev. C 72, 048801 (2005).

[14] N. Buyukcizmeci, R. Ogul, and A. S. Botvina, Eur. Phys. J. A 25, 57 (2005).

[15] S. Pratt, W. Bauer, C. Morling, and P. Underhill, Phys. Rev. C 63, 034608 (2000).

[16] C. Ishizuka, A. Ohnishi, and K. Sumiyoshi, Nucl. Phys. A 723, 517 (2003).

[17] S. Hudan et al., Phys. Rev. C 67, 064613 (2003).

[18] J. Iglio et al., Phys. Rev. C 74, 024605 (2006).

[19] D. V. Shetty et al., Phys. Rev. C 71, 024602 (2005).

[20] A. LeFèvre et al., Phys. Rev. Lett. 94, 162701 (2005).

[21] D. Henzlova et al., nucl-ex/0507003 (2005).

[22] G. A. Souliotis et al., Phys. Rev. C 73, 024606 (2006).

[23] M. A. Famiano et al., Phys. Rev. Lett. 97, 052701 (2006).

[24] D. V. Shetty, S. J. Yennello, and G. A. Souliotis, Phys. Rev. C, submitted (nucl-ex/0505011) (2006).

[25] G. A. Souliotis et al., Phys. Rev. Lett. 91, 022701 (2003).

[26] G. A. Souliotis et al., Phys. Lett. B 543, 163 (2002).

[27] L. Tassan-Got and C. Stefan, Nucl. Phys. A 524, 121 (1991).

[28] M. Veselsky et al., Phys. Rev. C 62, 064613 (2000).

[29] M. Veselsky et al., Nucl. Phys. A 724, 431 (2003).

[30] G. A. Souliotis, M. Veselsky, D. V. Shetty, and S. Y. Yennello, Phys. Lett. B 588, 35 (2004).

[31] A. S. Botvina and I. N. Mishustin, Phys. Rev. C 63, 061601 (2001).

[32] R. Charity, Phys. Rev. C 58, 1073 (1998).

[33] A. S. Botvina and I. N. Mishustin, nucl-th/0510081 (2005).

[34] M. Beyer, S. Strauss, P. Schuck, and S. A. Sofianos, Eur. Phys. J. A 22, 261 (2004).

[35] P. Moller, J. R. Nix, and K. L. Kratz, At. Data Nucl. Data Tables 66, 131 (1997). 